\title{
Eine Fehlerquelle bei der Bestimmung von Phosphorsäure mit Magnesia-Mixtur. \\ Von
}

Dr. N. v. Lorenz.

(Mittheilung der K. K. landw. ehemisehen Versuchsstation in Wien.)

Bei Versuchen, welche den Zweck hatten festzustellen, mit welcher Genauigkeit sich die Phosphorsäure durch tropfenweisen Zusatz von Magnesia-Mixtur - bei Abwesenheit, sowie in Gegenwart variirender Mengen von Citronensäure - bestimmen lasse, wurde die Beobachtung gemacht, dass die Menge des schliesslich ausgewogenen Magnesimpyrophosphates stets um einige wenige Milligramme geringer ausfiel, wenn auch nur ein halbes Procent Citronensäure in der Phosphorsä́urelösung zugegen war, als wenn gar keine Citronensäure vorhanden war. Es lag der Gedanke nahe, dass auch bei tropfenweisem Zusatz von MagnesiaMixtur zu einer citronensäurefreien. Phosphorsäurelösung eine geringe Menge von Magnesiumhydroxyd in das gefällte Magnesiumammoniumphosphat eingeht. Die Richtigkeit dieser Annahme wurde in unzweifelhafter Weise festgestellt, wie die folgenden Versuche beweisen.

Ein grösseres Quantum chemisch reines Mono-Ammoniumphosphat $\left(\mathrm{NH}_{4}\right) \mathrm{H}_{2} \mathrm{PO}_{4}$ wurde in Wasser gelöst; davon wurden fünfmal je $50 \mathrm{cc}$ in 5 Bechergläser abpipettirt, je $100 \mathrm{cc} 2$ procentige Ammoniakflüssigkeit zugesetzt und hierauf die Fällung der Phosphorsäure durch tropfenweisen Zusatz von 25 ce vorschriftsmässiger Magnesia-Mixtur bewerkstelligt. Die Niederschläge wurden auf 5 Schleicher-Schüll-Filtern je 10 mal mit 2 procentiger Ammoniakflüssigkeit gewaschen und ergaben nach dem Glühen. bis zur blendenden Weisse folgende fünf Netto-Gewichte:

$$
\begin{array}{ll} 
& 0,3310 \mathrm{~g} \\
& 0,3310 \ll \\
& 0,3310 \ll \\
& 0,3315 \ll \\
& 0,3315 \ll \\
\text { In Summa . . } \quad . \quad 1,6560 \mathrm{~g} \mathrm{Mg}_{2} \mathrm{P}_{2} & \mathrm{O}_{7}, \\
\text { entsprechend . . . } 1,0593 \ll \mathrm{P}_{2} \mathrm{O}_{5} .
\end{array}
$$

Ausserdem wurden gleichzeitig in einer neuen Platinschale $5 g$ reines Zinkoxyd auf dem Gebläse bis zur Gewichtsconstanz und hierauf noch 
eine halbe Stunde lang geglüht und dann gewogen. Nun wurden in der etwa $100 \mathrm{cc}$ fassenden Platinschale mit dem Zinkoxyd allmählich $5 \times 50=250 c c$ derselben Lösung von Monoammoniumphosphat auf dem Wasserbade (destillirtes Wasser) zur Trockne verdampft, im Trockenschranke getrocknet, einige Minuten über einem Drahtnetze schwach erhitzt (ohne dass dunkie Rothgluth auch nur an der äusseren Schalenfläche anftràt) und gewogen. Dann wurde die Schale 5 Minuten lang beim heftigsten Gebläsefeuer geglüht und abermals gewogen. Das Gewicht blieb constant beim schwachen Erhitzen und beim starken Glühen, und ergaben sich jedesmal $1,0495 g$, welche direct als $\mathrm{P}_{2} \mathrm{O}_{5}$ anzusprechen sind.

Es wurden nun von derselben Lösung neuerdings 5 Fällungen à $50 \mathrm{cc}$ gemacht und nochmals $250 \mathrm{cc}$ mit Zinkoxyd verdampft. Durch die Fällungen wurden erhalten:

$\begin{array}{ll} & 0,3309 \mathrm{~g} \\ & 0,3313 \ll \\ & 0,3313 \ll \\ & 0,3314 \ll \\ & \\ & 0,3316 \ll \\ \text { In Snmma } \cdot \quad \cdot \quad 1,6565 \mathrm{~g} \mathrm{Mg} \\ \text { entsprechend } \quad \cdot \quad 1,0595 \ll \mathrm{P}_{2} \mathrm{O}_{5} .\end{array}$

Die zweite Abdampfung mit Zinkoxyd ergab nach Einhaltung der vorhin angegebenen Operationen $1,0505 g \mathrm{P}_{2} \mathrm{O}_{\overline{5}}$.

Addirt man die correspondirenden Zahlen der ersten und zweiten Versuchsreihe, so ergibt sich:

Durch directes Abdampfen mit Zinkoxyd wurden erhalten:

$$
\text { entsprechend } \begin{aligned}
& 2,1000 g_{2} \mathrm{P}_{2} \mathrm{O}_{5} \\
& 3,2833 g \mathrm{Mg}_{2} \mathrm{P}_{2} \mathrm{O}_{7} .
\end{aligned}
$$

Durch Fällung mit Magnesia-Mixtur wurden dagegen erhalten (in Summa der 10 Bestimmungen):

$$
3,3125 g \mathrm{Mg}_{2} \mathrm{P}_{2} \mathrm{O}_{7} \text {. }
$$

Die Differenz zwischen den beiden letztgenannten Mengen von Magnesiumpyrophosphat beträgt somit $0,0292 \mathrm{~g}$.

Wenn die Ansicht richtig war, dass durch die Fällung mit tropfenweise zugesetzter Magnesia-Mixtur etwas Magnesia in das Magnesiumpyrophosphat eingeht, so sind obige $0,0292 \mathrm{~g}$ eben jener Mehrgehalt der 10 Niederschläge an Magnesia, und daher direct als solche anzusprechen. Unter diesen Verhältnissen enthält somit ein Niederschlag 
im Gewichte von etwa $330 \mathrm{mg}$ Magnesiumpyrophosphat nahezu $3 \mathrm{mg}$ oder nahezu 1\% Magnesia. Dass diese $3 \mathrm{mg}$ in der That Magnesia sind und nichts anderes, lässt sich am schlagendsten auf folgende Art beweisen. Wenn das gefällte Anmoniummagnesiumphosphat noch magnesiahaltig ist, so muss das Gewicht dieses Niederschlages sich entsprechend erhöhen, wenn man denselben nach dem Auswaschen mit Ammoniakflüssigkeit in Salzsäure auflöst, einige Tropfen Phosphorsäure hinzubringt und nun neuerdings mit Ammoniak fällt. Dieser Versuch wurde mit derselben Lösung von Monoammoniumphosphat ansgeführt, welche für die bisherigen Versuche verwendet worden war.

Die Resultate sind folgende: Gewicht des Magnesiumpyrophosphates erhalten durch Auflösen des gewaschenen Ammoniummagnesiumphosphates in Salzsäure, Zusatz von $20 \mathrm{mg}$ Phosphorsäureanhydrid $\left(\mathrm{P}_{2} \mathrm{O}_{5}\right)$ und erneuertes Fällen und Auswaschen mit Ammoniakflüssigkeit:

$$
\begin{array}{ll} 
& 0,3355 g \\
& 0,3360 \ll \\
& 0,3365 \ll \\
\text { Mittel } \quad \cdot \quad 0,3360 \mathrm{~g} \mathrm{Mg} & \mathrm{P}_{2} \mathrm{O}_{7} .
\end{array}
$$

Das Mittel aus den vorhin angeführten 10 directen Fällungen beträgt:

$$
0,3312 g \mathrm{Mg}_{2} \mathrm{P}_{2} \mathrm{O}_{7} \text {. }
$$

Die Differenz der zwei letztgenannten Mengen Magnesimpyrophosphat beträgt $0,0048 \mathrm{~g}$. Diese $4,8 \mathrm{mg}$ müssen Phosphorsäure $\left(\mathrm{P}_{2} \mathrm{O}_{5}\right)$ sein, welche nach Zusatz der $20 \mathrm{mg}$ Phosphorsäure durch die $3 \mathrm{mg}$ Magnesia gebunden und dann durch das Ammoniak mit niedergeschlagen wurden. Nun binden $3 \mathrm{mg}$ Magnesia thatsächlich $5,3 \mathrm{mg}$ Phosphorsäureanhydrid im Pyrophosphate, was mit unseren gefundenen $4,8 \mathrm{mg}$ hinreichend übereinstimmt.

Das richtige Gewicht dieser Niederschläge, wie es sich aus der durch Abdampfen der Ammonphosphatlösung mit Zinkoxyd erhaltenen Phosphorsäure berechnen lässt, wäre gewesen: $0,3283 \mathrm{~g} \mathrm{Mg}_{2} \mathrm{P}_{2} \mathrm{O}_{7}$.

Wenn die. Anwesenheit von Citronensäure beim Fällen der Niederschläge von Ammoniummagnesiumphosphat die Mitfällung von Magnesia verhindert, so muss unter Citronensäurezusatz gefälltes Magnesiumammoniumphosphat sein Gewicht constant erhalten, wenn es in Salzsäure gelöst, mit Phosphorsäure versetzt und wieder mit Ammoniak gefällt wird. Dass dies der Fall ist, beweisen die folgenden Zahlen. Sechsmal $50 \mathrm{cc}$ unserer Normallösung von Monoammoniumphosphat wurden in sechs Bechergläsern mit 100 cc 2 procentiger Ammoniakflüssigkeit und 
$2 \%$ Citronensäure (in Form der üblichen Ammoncitratlösung) versetzt, tropfenweise mit Magnesia-Mixtur gefällt, filtrirt und gewaschen. Drei dieser Niederschläge wurden direct geglüht, die drei anderen jedoch erst wie vorhin mit Salzsäure, Phosphorsäure und Ammoniak behandelt und dann weiss geglüht.

Die Gewichte dieser sechs Niederschläge waren:

A.

direct geglüht

$0,3280 \mathrm{~g}$

$0,3285 *$

$0,3285 *$

B.

vorher mit $\mathrm{HCl}, \mathrm{P}_{2} \mathrm{O}_{5}$ und $\mathrm{NH}_{3}$ behandelt

$0,3280 \mathrm{~g}$

0,3280 «

$0,3285 \ll$,

zum Beweise, dass hier kein Magnesiumoxyd in das Magnesiumammoniumphosphat übergegangen war. Aus dem angeführten folgt die Regel, dass man präcise Phosphorsäurebestimmungen mit Magnesia-Mixtur ausführen kann, wenn man der Phosphorsäurelösung ca. $2 \%$ - oder mehr Citronensäure in Form von Ammoncitratlösung zusetzt und dann die Magnesia-Mixtur aus einer Pipette ${ }^{1}$ ) mit enger Ausflussöffnung unter tüchtigem Umrühren zufliessen lässt.

Dagegen lässt sich in Abwesenheit von Citronensäure die Mitfällung von ein wenig Magnesia (wenigstens in stark ammoniakalischen Flüssigkeiten) auch beim langsamsten Zutröpfeln der Mixtur absolut nicht vermeiden.

W i e n, im November 1892.

\section{Bericht über die Fortschritte der analytischen Chemie.}

I. Allgemeine analytische Methoden, analytische Operationen, Apparate und Reagentien.

Von

W. Fresenius, unter Mitwirkung von W. Schranz.

Auf einige kürzlich erschienene Werke über analytische Chemie wollen wir nicht verfehlen die Leser dieser Zeitschrift hinzuweisen.

1) Wenn eine Phosphorsäurelösung $50 c c$ oder noch mehr der üblichen Ammoncitratlösung enthält, so ist das langsame Eintröpfeln der MagnesiaMixtur überflüssig. 